\title{
Vector and Axial Form Factors Applied to Neutrino Quasielastic Scattering
}

\author{
H. Budd ${ }^{\mathrm{a}}$, A. Bodek ${ }^{\mathrm{a}}$ and J. Arrington ${ }^{\mathrm{b}}$ \\ a Department of Physics and Astronomy, University of Rochester, Rochester, New York 14618, USA \\ b Argonne National Laboratory, Argonne, Illinois 60439, USA
}

We calculate the quasielastic cross sections for neutrino scattering on nucleons using up to date fits to the nucleon elastic electromagnetic form factors $G_{E}^{p}, G_{E}^{n}, G_{M}^{p}, G_{M}^{n}$, and weak form factors. We show the extraction of $F_{A}\left(q^{2}\right)$ for neutrino experiments. We show how well MINER $\nu \mathrm{A}$, a new approved experiment at FNAL, can measure $F_{A}\left(q^{2}\right)$. We show the that $F_{A}\left(q^{2}\right)$ has a different contribution to the anti-neutrino cross section, and how the anti-neutrino data can be used to check $F_{A}\left(q^{2}\right)$ extracted from neutrino scattering. (Presented by Howard Budd at NuInt04, Mar. 2004, Laboratori Nazionali del Gran Sasso - INFN - Assergi, Italy [1])

\section{INTRODUCTION}

Experimental evidence for oscillations among the three neutrino generations has been recently reported [2]. Since quasielastic (QE) scattering forms an important component of neutrino scattering at low energies, we have undertaken to investigate $\mathrm{QE}$ neutrino scattering using the latest information on nucleon form factors.

Recent experiments at SLAC and Jefferson Lab (JLab) have given precise measurements of the vector electromagnetic form factors for the proton and neutron. These form factors can be related to the form factors for QE neutrino scattering by conserved vector current hypothesis, CVC. These more recent form factors can be used to give better predictions for QE neutrino scattering and better determination of the axial form factor, $F_{A}\left(q^{2}\right)$.

\section{EQUATIONS FOR QE SCATTERING}

The hadronic current for QE neutrino scattering is given by [3]

$$
\begin{aligned}
& <p\left(p_{2}\right)\left|J_{\lambda}^{+}\right| n\left(p_{1}\right)>= \\
& \bar{u}\left(p_{2}\right)\left[\gamma_{\lambda} F_{V}^{1}\left(q^{2}\right)+\frac{i \sigma_{\lambda \nu} q^{\nu} \xi F_{V}^{2}\left(q^{2}\right)}{2 M}\right. \\
& \left.\quad+\gamma_{\lambda} \gamma_{5} F_{A}\left(q^{2}\right)+\frac{q_{\lambda} \gamma_{5} F_{P}\left(q^{2}\right)}{M}\right] u\left(p_{1}\right),
\end{aligned}
$$

where $q=k_{\nu}-k_{\mu}, \xi=\left(\mu_{p}-1\right)-\mu_{n}$, and $M=$ $\left(m_{p}+m_{n}\right) / 2$. Here, $\mu_{p}$ and $\mu_{n}$ are the proton and neutron magnetic moments. We assume that there are no second class currents, so the scalar form factor $F_{V}^{3}$ and the tensor form factor $F_{A}^{3}$ need not be included.

The form factors $F_{V}^{1}\left(q^{2}\right)$ and $\xi F_{V}^{2}\left(q^{2}\right)$ are given by:

$$
\begin{gathered}
F_{V}^{1}\left(q^{2}\right)=\frac{G_{E}^{V}\left(q^{2}\right)-\frac{q^{2}}{4 M^{2}} G_{M}^{V}\left(q^{2}\right)}{1-\frac{q^{2}}{4 M^{2}}}, \\
\xi F_{V}^{2}\left(q^{2}\right)=\frac{G_{M}^{V}\left(q^{2}\right)-G_{E}^{V}\left(q^{2}\right)}{1-\frac{q^{2}}{4 M^{2}}} .
\end{gathered}
$$

We use the CVC to determine $G_{E}^{V}\left(q^{2}\right)$ and $G_{M}^{V}\left(q^{2}\right)$ from the electron scattering form factors $G_{E}^{p}\left(q^{2}\right), G_{E}^{n}\left(q^{2}\right), G_{M}^{p}\left(q^{2}\right)$, and $G_{M}^{n}\left(q^{2}\right)$ :

$$
\begin{aligned}
G_{E}^{V}\left(q^{2}\right) & =G_{E}^{p}\left(q^{2}\right)-G_{E}^{n}\left(q^{2}\right), \\
G_{M}^{V}\left(q^{2}\right) & =G_{M}^{p}\left(q^{2}\right)-G_{M}^{n}\left(q^{2}\right) .
\end{aligned}
$$

Previously, many neutrino experiment have assumed that the vector form factors are described by the dipole approximation.

$$
G_{D}\left(q^{2}\right)=\frac{1}{\left(1-\frac{q^{2}}{M_{V}^{2}}\right)^{2}}, \quad M_{V}^{2}=0.71 \mathrm{GeV}^{2}
$$




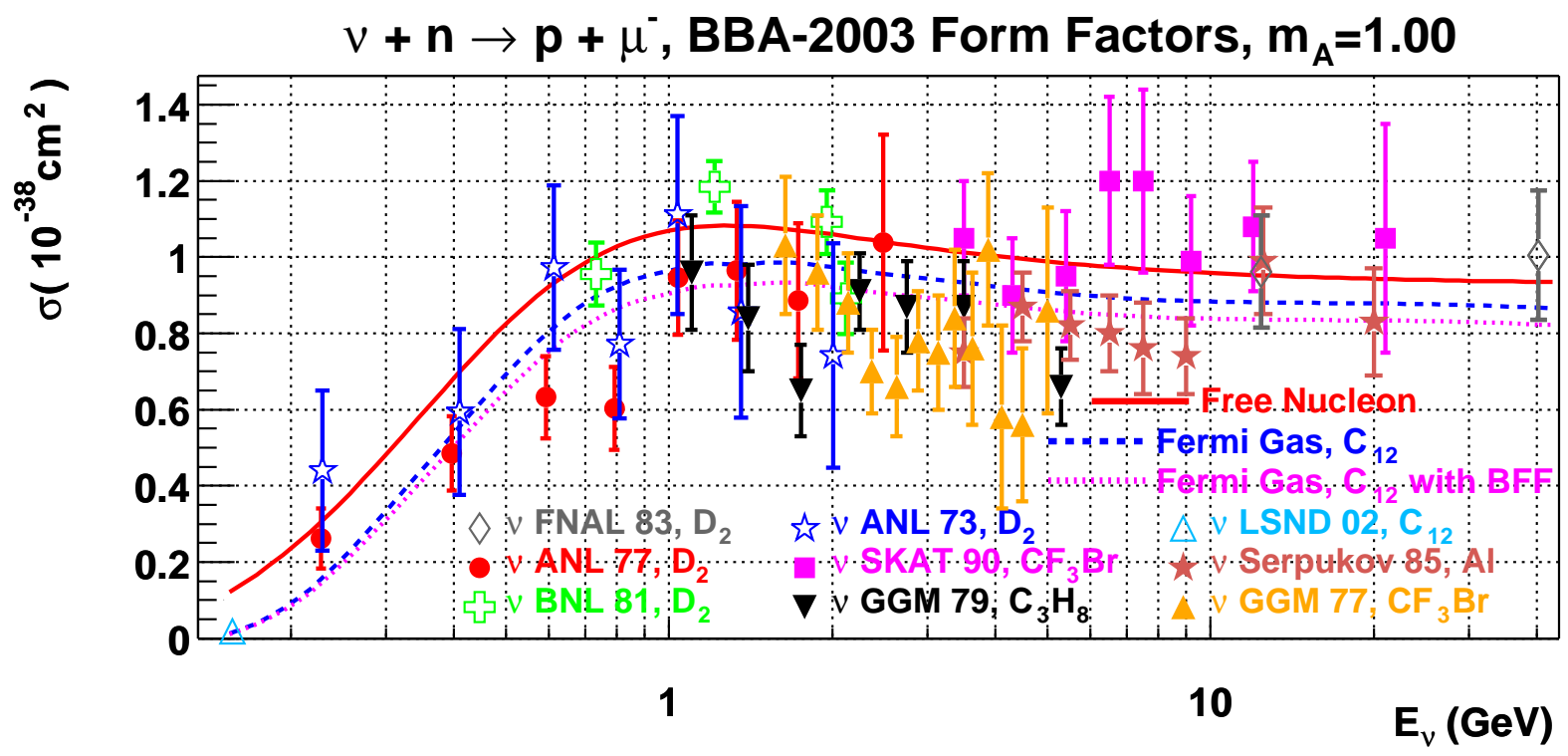

Figure 1. The QE neutrino cross section along with data from various experiments. The calculation uses $M_{A}=1.00 \mathrm{GeV}, g_{A}=-1.267, M_{V}^{2}=0.71 \mathrm{GeV}^{2}$ and BBA-2003 Form Factors. The solid curve uses no nuclear correction, while the dashed curve [7] uses a Fermi gas model for carbon with a $25 \mathrm{MeV}$ binding energy and 220 Fermi momentum. The dotted curve is the prediction for carbon including both Fermi gas Pauli blocking and the effect of nuclear binding on the nucleon form factors [10] (bounded form factors). The data shown are from FNAL 1983 [11, ANL 1977 [12], BNL 1981 [13], ANL 1973 [14], SKAT 1990 [15], GGM 1979 [16], LSND 2002 [17, Serpukov 1985 [18], and GGM 1977 [19].

$$
\begin{gathered}
G_{E}^{p}=G_{D}\left(q^{2}\right), \quad G_{E}^{n}=0, \\
G_{M}^{p}=\mu_{p} G_{D}\left(q^{2}\right), \quad G_{M}^{n}=\mu_{n} G_{D}\left(q^{2}\right) .
\end{gathered}
$$

We refer to the above combination of form factors as 'Dipole Form Factors'. It is an approximation that has been improved by us in a previous publication [4. We use our updated form factors which we refer as 'BBA-2003 Form Factors' 4] [5] (Budd, Bodek, Arrington).

The axial form factor is given by

$$
F_{A}\left(q^{2}\right)=\frac{g_{A}}{\left(1-\frac{q^{2}}{M_{A}^{2}}\right)^{2}} .
$$

We have used our updated value of $M_{A}=1.00 \pm$ $0.020 \mathrm{GeV}$ [4 which is in good agreement with the theoretically corrected value from pion electroproduction of $1.014 \pm 0.016 \mathrm{GeV}$ [6]. For extraction of $F_{A}\left(q^{2}\right)$ we use the value of $M_{A}=$ 1.014 , since it is independent of QE scattering measurements.

\section{Comparison to Cross Section Data}

Figures 1 shows the QE cross section for $\nu$ using BBA-2003 Form Factors and $M_{A}=1.00 \mathrm{GeV}$ The normalization uncertainty in the data is approximately $10 \%$. The solid curve uses no nuclear correction, while the dotted curve [7] uses a NUANCE [8] calculation of a Smith and Moniz 9] based Fermi gas model for carbon. This nuclear model includes Pauli blocking and Fermi motion, but not final state interactions. The Fermi gas model was run with a $25 \mathrm{MeV}$ binding energy and $220 \mathrm{MeV}$ Fermi momentum. The dotted curve is the prediction for carbon including both Fermi gas Pauli blocking and the effect of nuclear binding on the nucleon form factors as modeled by Tsushima et al. [10. The ratio of bounded form factors to free form factors is set to 1 for $Q^{2}>2.0 \mathrm{GeV}$. The updated form factors improve the agreement with neutrino QE cross section data and give a reasonable description of the 
QE, $\nu_{\mu}, \Delta\left(\mathrm{d} \sigma / \mathrm{dQ} Q^{2}\right)[\%]$ for $1 \%$ Change in $\mathrm{FF}, \mathrm{M}_{\mathrm{A}}=1$

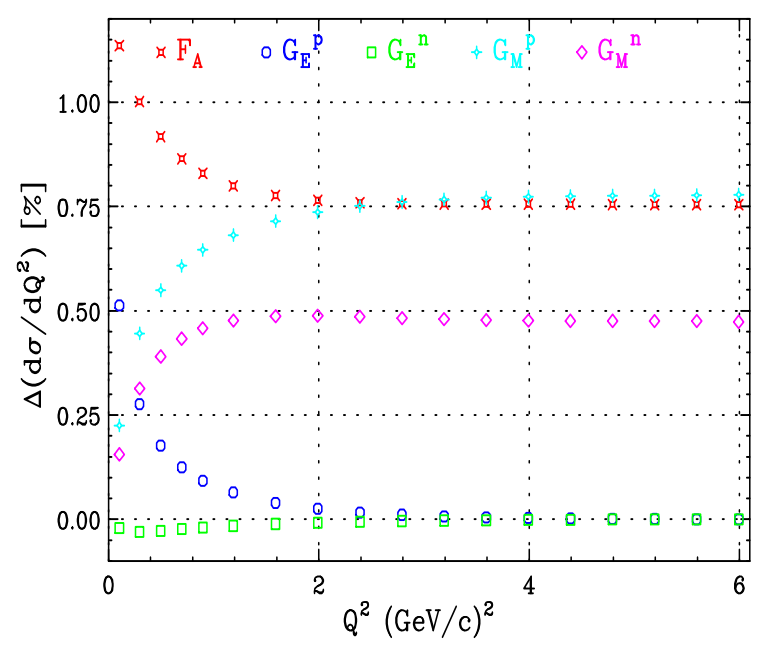

Figure 2. The percent change in the neutrino cross section for a $1 \%$ change in the form factors.

cross sections from deuterium. We plan to study the nuclear corrections, adopting models which have been used in precision electron scattering measurements from nuclei at SLAC and JLab.

\section{Extraction of $F_{A}\left(q^{2}\right)$}

A substantial fraction of the cross section comes from the form factor $F_{A}\left(q^{2}\right)$. Therefore, we can extract $F_{A}\left(q^{2}\right)$ from the differential cross section. Figure 2 and 3 show the contribution of $F_{A}\left(q^{2}\right)$ to $d \sigma / d Q^{2}$. Figure 2 shows the percent change in the neutrino cross section for a $1 \%$ change in the form factors. Figure 3 shows the fractional contribution of the form factor determined by setting the form factor to zero and by determining the fractional decrease in the differential cross section. Since some terms are products of different form factors, the sum of the curves do not have be 1 .

To extract $F_{A}$, we write the equation for $d \sigma / d q^{2}\left(q^{2}, E_{\nu}\right)$ in terms of a quadratic function of $F_{A}\left(q^{2}\right)$.

$$
\begin{aligned}
a\left(q^{2}, E_{\nu}\right) F_{A}\left(q^{2}\right)^{2}+ & b\left(q^{2}, E_{\nu}\right) F_{A}\left(q^{2}\right) \\
& +c\left(q^{2}, E_{\nu}\right)-\frac{d \sigma}{d q^{2}}\left(q^{2}, E_{\nu}\right)=0
\end{aligned}
$$

$\mathrm{QE}, \nu_{\mu}$, Form Factor contribution, $\mathrm{M}_{\mathrm{A}}=1$



Figure 3. Fractional contribution of the form factor determined by setting the form factor to zero and by determining the fractional decrease in the differential cross section, 1 $\left(d \sigma / d Q^{2}(\right.$ formfactor $\left.=0)\right) /\left(d \sigma / d Q^{2}\right)$.

For each $q^{2}$ bin, we integrate the above equation over the $q^{2}$ bin and the neutrino flux.

$$
\begin{array}{r}
\iint d q^{2} d E_{\nu}\left\{a\left(q^{2}, E_{\nu}\right) F_{A}\left(q^{2}\right)^{2}+b\left(q^{2}, E_{\nu}\right) F_{A}\left(q^{2}\right)\right. \\
\left.+c\left(q^{2}, E_{\nu}\right)-\frac{d \sigma}{d q^{2}}\left(q^{2}, E_{\nu}\right)\right\}=0
\end{array}
$$

The above equation can be written as a quadratic equation in $F_{A}$ at the bin value $q_{b i n}^{2}$.

$$
\alpha F_{A}\left(q_{b i n}^{2}\right)^{2}+\beta F_{A}\left(q_{b i n}^{2}\right)+\gamma-\Delta-N_{B i n}^{D a t a}=0
$$

The terms of this equation are given below:

$$
\begin{aligned}
& \alpha=\iint d q^{2} d E_{\nu} a\left(q^{2}, E_{\nu}\right) \\
& \beta=\iint d q^{2} d E_{\nu} b\left(q^{2}, E_{\nu}\right) \\
& \gamma=\iint d q^{2} d E_{\nu} c\left(q^{2}, E_{\nu}\right)
\end{aligned}
$$




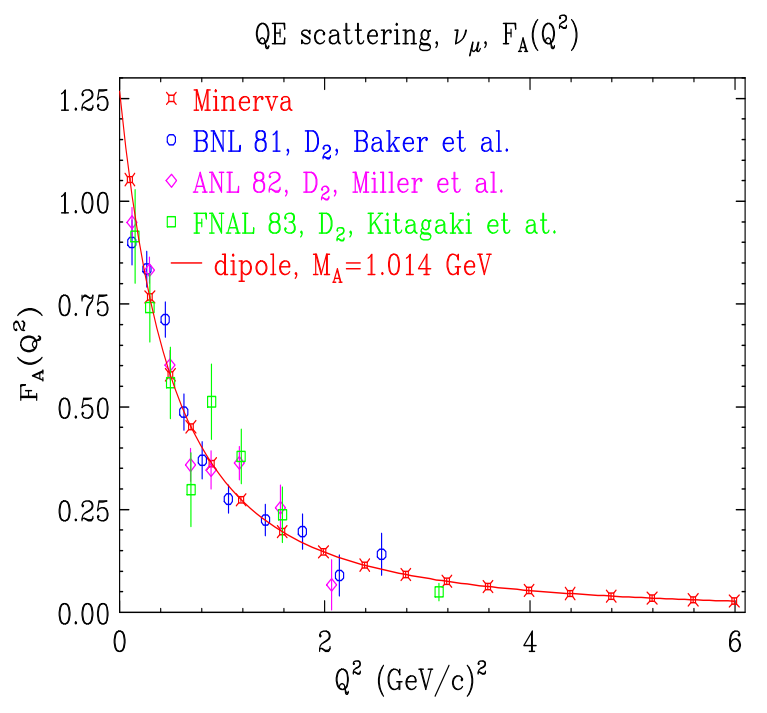

Figure 4. Extracted values of $F_{A}\left(q^{2}\right)$ for the three deuterium bubble chamber experiments Baker et al. [13, Miller et al. 21], and Kitagaki et al. 11. Also shown are the expected errors for MINER $\nu \mathrm{A}$ assuming a dipole form factor for $F_{A}\left(q^{2}\right)$ with $\mathrm{M}_{A}=1.014$.

To find $q_{b i n}^{2}$, we assume a nominal $F_{A}\left(q^{2}\right)$, written $F_{A}^{N}\left(q^{2}\right)$. We determine $q_{b i n}^{2}$ from

$\alpha F_{A}^{N}\left(q_{b i n}^{2}\right)^{2}-\iint d q^{2} d E_{\nu} a\left(q^{2}, E_{\nu}\right) F_{A}^{N}\left(q^{2}\right)^{2}=0$.

$\Delta$ is a bin center correction term which also uses $F_{A}^{N}\left(q^{2}\right) . \Delta$ is determined by

$\Delta=\beta F_{A}^{N}\left(q_{b i n}^{2}\right)-\iint d q^{2} d E_{\nu} b\left(q^{2}, E_{\nu}\right) F_{A}^{N}\left(q^{2}\right)$.

The number of events in the bin is given by $N_{\text {Bin }}^{\text {Data }}$. The number of events in the bin from theory is

$N_{B i n}^{T h y}=\iint d q^{2} d E_{\nu} \frac{d \sigma}{d q^{2}}\left(q^{2}, E_{\nu}\right)$.

The errors in the points are given by

$\frac{\sqrt{N_{B i n}^{T h y}}}{2 \alpha F_{A}^{N}\left(q_{b i n}^{2}\right)+\beta}$.



Figure 5. Same as Figure 4 with a logarithmic scale.

Figure 4 and 5 show our extracted values of $F_{A}\left(q^{2}\right)$ for the three deuterium bubble chamber experiments. For these plots the curve shown in the figures is a dipole with $m_{A}=1.014$, the value extracted from pion-electro production. The data and fluxes given in their papers are used in the extraction of $F_{A}\left(q^{2}\right)$. These plots show the previous data is not sufficient to determine the form for $F_{A}\left(q^{2}\right)$.

In addition, we have shown the expected values for MINER $\nu \mathrm{A}$ and its errors. We have plotted MINER $\nu \mathrm{A}$ assuming it is a dipole. We have assumed a 4 year run with 3 tons of fiducial volume and included the effects of inefficiencies and backgrounds. Resolution smearing and systematic errors are not included.

Figure [ plots $F_{A}\left(q^{2}\right) /$ dipole to show how well MINER $\nu \mathrm{A}$ can measure $F_{A}\left(q^{2}\right) . \quad G_{E}^{p}\left(q^{2}\right)$ from electron scattering experiments depends upon the measuring technique [5]. For MINER $\nu \mathrm{A}$ we show $F_{A}$ under the assumption $F_{A}\left(q^{2}\right) /$ dipole $=G_{E}^{p}\left(q^{2}\right) /$ dipole from the cross section technique (Rosenbuth separation) and $F_{A}\left(q^{2}\right) /$ dipole $=G_{E}^{p}\left(q^{2}\right) /$ dipole from polarization transfer technique. The MINER $\nu \mathrm{A}$ errors are plotted assum- 
QE scattering, $\nu_{\mu}, \mathrm{F}_{\mathrm{A}}\left(\mathrm{Q}^{2}\right) /$ dipole, $\mathrm{M}_{\mathrm{A}}=1.014 \mathrm{GeV}$

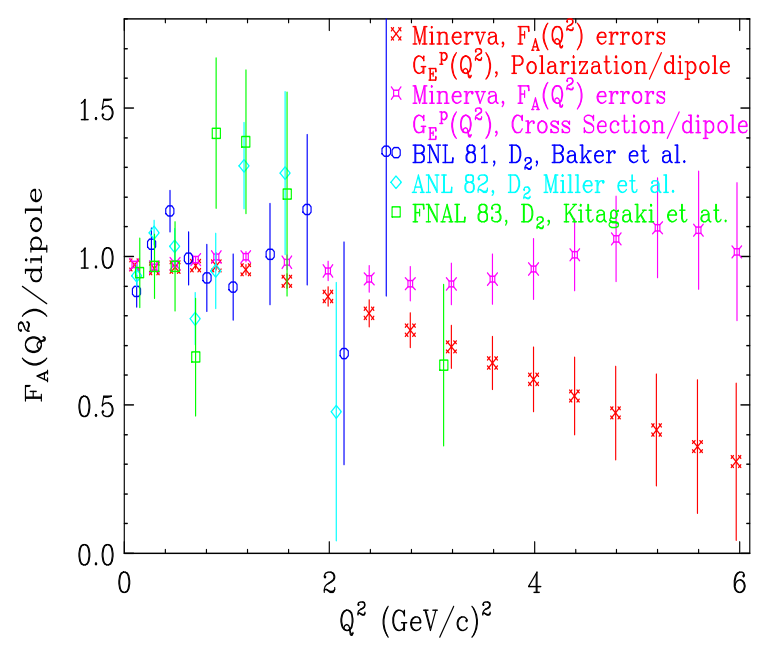

Figure 6. Extracted values of $F_{A}\left(q^{2}\right) /$ dipole for deuterium bubble chamber experiments Baker et al. 13, Miller et al. 21], and Kitagaki et al. 11]. For MINER $\nu \mathrm{A}$ the projected results are shown for two different assumptions: $F_{A} /$ dipole $=G_{E}^{p} /$ dipole from cross section and $F_{A} /$ dipole $=G_{E}^{p} /$ dipole from polarization. The MINER $\nu \mathrm{A}$ errors are for a 4 year run.

ing the plotted $F_{A}\left(q^{2}\right)$ is the nominal $F_{A}\left(q^{2}\right)$. We see that the measurement of $F_{A}\left(q^{2}\right)$ from MINER $\nu \mathrm{A}$ can distinguish between these to the two possible forms. In addition, MINER $\nu \mathrm{A}$ can determine whether $F_{A}\left(q^{2}\right)$ is a dipole or not.

\section{Extraction of $F_{A}\left(q^{2}\right)$ from anti-neutrinos}

The determination of $F_{A}\left(q^{2}\right)$ will have systematic errors from the flux, nuclear effects, QE identifications, background determination, etc. Antineutrino data can provide a check on $F_{A}\left(q^{2}\right)$. Figure 7 and 8 show the contribution of $F_{A}\left(q^{2}\right)$ to the cross section vs $Q^{2}$ for anti-neutrinos. Figure $\mathbf{7}$ shows the percent change in the anti-neutrino cross section for a $1 \%$ change in the form factors. The plot shows that $F_{A}\left(q^{2}\right)$ has a different contribution to the cross section for anti-neutrinos than neutrinos. At $Q^{2} \sim 3 G e V^{2}, F_{A}$ is not con-
$\mathrm{QE}, \bar{\nu}_{\mu}, \Delta\left(\mathrm{d} \sigma / \mathrm{d} \mathrm{Q}^{2}\right)[\%]$ for $1 \%$ Change is $\mathrm{FF}, \mathrm{M}_{\mathrm{A}}=1$

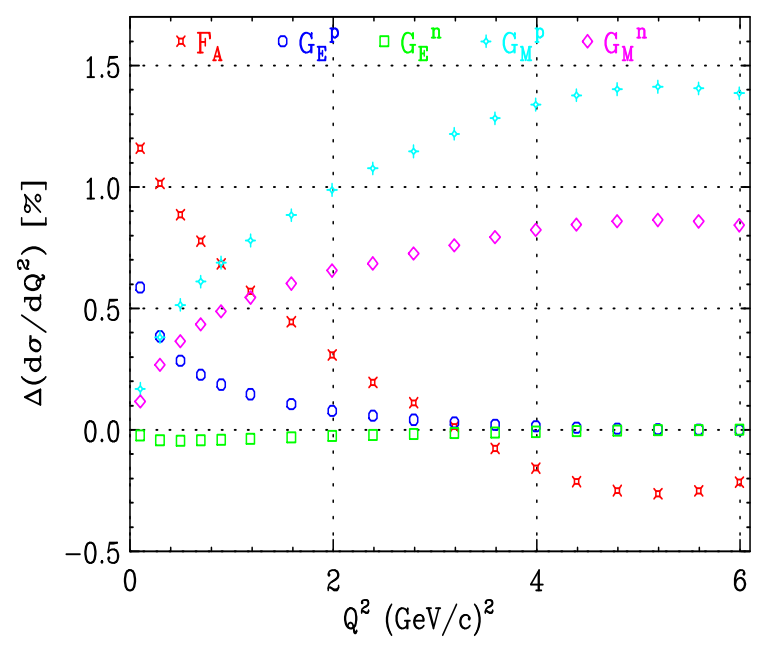

Figure 7. The percent change in the antineutrino cross section for a $1 \%$ change in the form factors.

tributing to the cross section, and the cross section becomes independent of $F_{A}\left(q^{2}\right)$. Hence, at higher $Q^{2}$ the cross section can be predicted and compared to the data to determine errors to the neutrino extraction. Figure 8 shows the fractional contribution of the form factor determined by setting the form factor to zero and by determining the fractional decrease in the differential cross section. Note, since some terms are products of different form factors the sum of the curves do not have to sum to 1 .

Figure 9] shows the errors on $F_{A}$ /dipole for antineutrinos. The overall errors scale is arbitrary. As we expect, the errors on $F_{A}\left(q^{2}\right)$ become large at $Q^{2}$ around $3 \mathrm{GeV}^{2}$ when the derivative of the cross section with respect to $F_{A}\left(q^{2}\right)$ goes to 0 .

\section{Conclusions}

We have used new form factors to show the cross sections for QE neutrino scattering. The cross sections give a reasonable description of the deuterium data, but the nuclear data is low. We have shown how to extract $F_{A}$ and have shown show well MINER $\nu \mathrm{A}$ can measure $F_{A}\left(q^{2}\right)$. For 


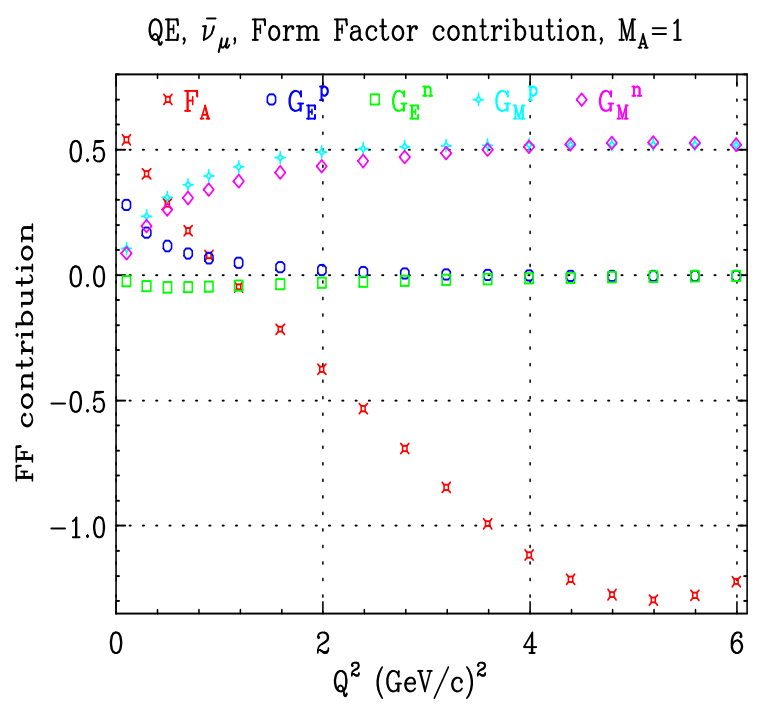

Figure 8. $1-d \sigma / d Q^{2}($ form factor $=0) / d \sigma / d Q^{2}$. The contribution of the form factors determined by setting the form factors $=0$.

anti-neutrino data at high $Q^{2}, F_{A}$ has a different contribution, so anti-neutrinos provides a check for the extraction of $F_{A}$ from neutrinos.

\section{Acknowledgments}

This work is supported in part by the U. S. Department of Energy, Nuclear Physics Division, under contract W-31-109-ENG-38 (Argonne) and High Energy Physics Division under grant DEFG02-91ER40685 (Rochester).

\section{REFERENCES}

1. http://nuint04.lngs.infn.it/

2. Y. Fukada et al., Phys. Rev. Lett. 81 (1998) 1562.

3. C.H. Llewellyn Smith, Phys. Rep. 3C (1972).

4. H. Budd, A. Bodek and J. Arrington, hepex[0308005].

5. J. Arrington, Phys. Rev. C68 034325 (2003), nucl-ex[0305009]; J. Arrington, Phys. Rev. C69 022201 (2004).

6. V. Bernard, L. Elouadrhiri, U.G. Meissner, J.Phys.G28 (2002), hep-ph[0107088].

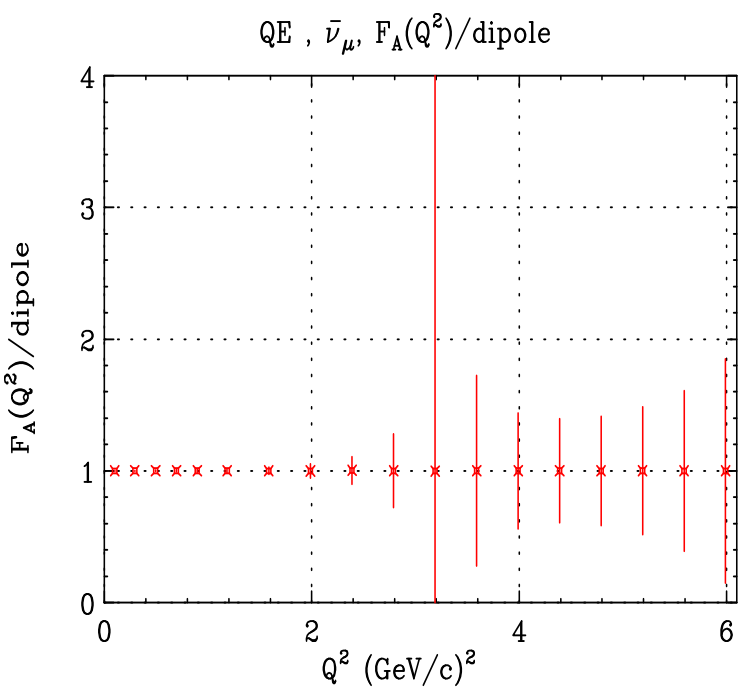

Figure 9. The relative errors for an extraction of $F_{A}$ using anti-neutrinos. The errors are shown for $F_{A}\left(Q^{2}\right) /$ dipole. The flux is arbitrary.

7. G. Zeller, private communication.

8. D. Casper, Nucl. Phys. Proc. Suppl. 112 (2002) 161.

9. R.A. Smith and E.J. Moniz, Nucl. Phys. B43 (1972) 605.

10. K. Tsushima, Hungchong Kim, K. Saito, nucl-th[0307013].

11. T. Kitagaki et al., Phys. Rev. D26 (1983) 436.

12. S.J. Barish et al., Phys. Rev. D16 (1977) 3103.

13. N.J. Baker et al., Phys. Rev. D23 (1981) 2499.

14. W.A. Mann et al., Phys. Rev. Lett. 31 (1973) 844.

15. J. Brunner et al., Z. Phys. C45 (1990) 551.

16. M. Pohl et al., Lett. Nuovo Cimento 26 (1979) 332.

17. L.B. Auerbach et al., Phys. Rev. C66 (2002) 015501.

18. S.V. Belikov et al., Z. Phys. A320 (1985) 625.

19. S. Bonetti et al., Nuovo Cimento 38 (1977) 260.

20. N. Armenise et al., Nucl. Phys. B152 (1979) 365.

21. K.L. Miller et al., Phys. Rev. D26 (1982) 537. 22. Minerva Collaboration, Proposal for Fermilab 
Experiment E938, hep-ex[0405002] 


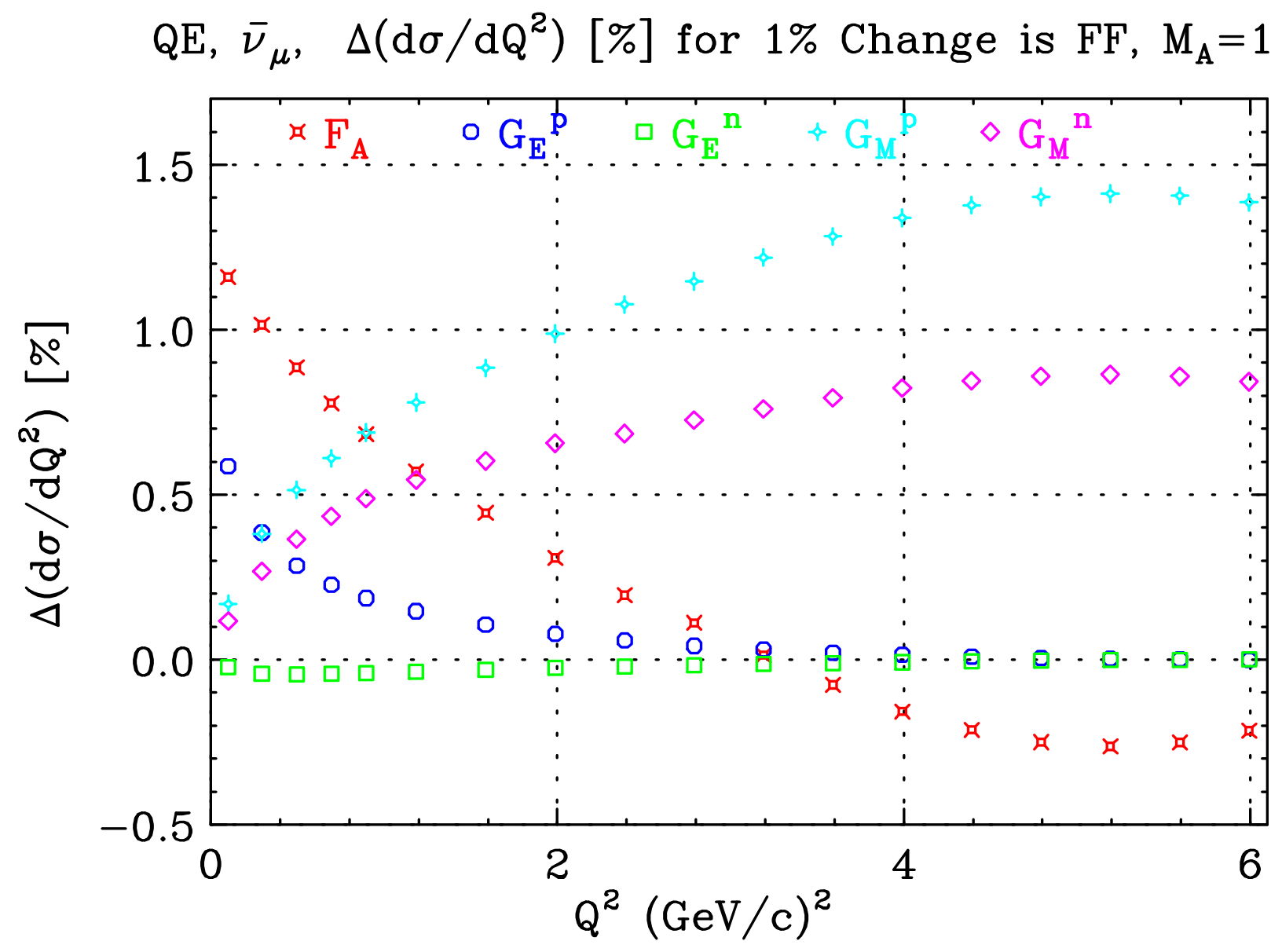

